DEPARTMENT OF ENVIRONMENT,

TECHNOLOGY AND TECHNOLOGY MANAGEMENT

\title{
D-optimal and D-efficient Equivalent-Estimation Second-Order Split-Plot Designs
}

\author{
Harrison Macharia \& Peter Goos
}

\section{UNI VERSI TY OF ANTWERP}

\section{Faculty of Applied Economics}

Stadscampus

Prinsstraat 13, B.213

BE-2000 Antwerpen

Tel. +32 (0)32654032

Fax +32 (0)3 2654799

http://www.ua.ac.be/tew 


\title{
FACULTY OF APPLIED ECONOMI CS
}

\author{
DEPARTMENT OF ENVIRONMENT, \\ TECHNOLOGY AND TECHNOLOGY MANAGEMENT \\ D-optimal and D-efficient Equivalent-Estimation \\ Second-Order Split-Plot Designs
}

Harrison Macharia \& Peter Goos

RESEARCH PAPER 2010-011

MAY 2010

University of Antwerp, City Campus, Prinsstraat 13, B-2000 Antwerp, Belgium Research Administration - room B.213

phone: (32) 32654032

fax: (32) 32654799

e-mail: joeri.nys@ua.ac.be

The papers can be also found at our website: www.ua.ac.be/ tew (research $>$ working papers) \& www.repec.org/ (Research papers in economics - REPEC)

D/ 2010/1169/011 


\title{
D-optimal and D-efficient Equivalent-Estimation Second-Order Split-Plot Designs
}

\author{
Harrison Macharia ${ }^{1}$ \\ Peter Goos ${ }^{1,2}$ \\ ${ }^{1}$ Faculty of Applied Economics, Universiteit Antwerpen, \\ Prinsstraat 13, 2000 Antwerpen, Belgium. \\ ${ }^{2}$ Erasmus School of Economics, Erasmus Universiteit Rotterdam, \\ Postbus 1738, 3000 DR Rotterdam, Netherlands.
}

May 28, 2010

\begin{abstract}
SUMMARY
Industrial experiments often involve factors which are hard to change or costly to manipulate and thus make it impossible to use a complete randomization. In such cases, the split-plot design structure is a cost-efficient alternative that reduces the number of independent settings of the hard-to-change factors. In general, model estimation for split-plot designs requires the use of generalized least squares (GLS). However, for some split-plot designs (including not only classical agricultural split-plot designs, but also some second-order split-plot response surface designs), ordinary least squares (OLS) estimates are equivalent to GLS estimates. These designs are called equivalent-estimation designs. As an alternative to these equivalentestimation designs, one can use D-optimal designs which guarantee efficient estimation of the fixed effects of the statistical model that is appropriate given the split-plot structure. We explore the relationship between equivalent-estimation and D-optimal split-plot designs for a second-order response surface model and propose an algorithm for generating D-efficient equivalent-estimation split-plot designs. This approach allows for a flexible choice of the number of hard-to-change factors, the number of easy-to-change factors, the number of whole plots and the total sample size.
\end{abstract}

Key Words: coordinate-exchange algorithm, D-optimality, equivalent estimation, generalized least squares, ordinary least squares, split-plot design. 


\section{Introduction}

Industrial experiments often involve factors that are hard to change, expensive or time-consuming to manipulate. These factors make complete randomization undesirable. In such cases, the split-plot design is an alternative that reduces the number of independent settings of the hardto-change factors and therefore the experimental cost. The remaining factors in the experiment, which are relatively less costly to manipulate, are referred to as easy-to-change factors.

Split-plot designs first gained popularity in agricultural experiments where large tracts of land were subdivided in relatively large portions known as whole plots. Each of the possible levels of the whole-plot factors was then assigned to these plots. Whole plots were further divided into smaller portions known as subplots or split-plots where subplot factors were applied. Thus whole-plot factors vary from whole plot to whole plot while subplot factors vary from subplot to subplot. In the context of industrial experiments, hard-to-change factors act as whole-plot factors, whereas the easy-to-change factors act as subplot factors.

The design and analysis of split-plot industrial experiments has received considerable attention in the literature in recent years. Letsinger, Myers and Lentner (1996) discussed response surface methods for split-plot designs focusing on the analysis of these designs. They recommended the use of generalized least squares (GLS) and restricted maximum likelihood (REML) for estimating split-plot response surface models.

Huang, Chen and Voelkel (1998), Bingham and Sitter (1999) and Bingham, Schoen and Sitter (2004) described the construction of two-level fractional factorial split-plot designs using the aberration criterion. Multistratum response surface designs, of which split-plot designs are special cases, are discussed in Trinca and Gilmour (2001). They present a sequential method for constructing these designs, from stratum to stratum and starting in the highest stratum. Kulahci and Bisgaard (2005) illustrated how split-plot designs can be constructed from Plackett-Burman designs. Goos and Vandebroek (2001, 2003, 2004) and Jones and Goos (2007) propose exchange algorithms for constructing D-optimal split-plot designs. A review of the recent developments on the design of split-plot experiments can be found in Jones and Nachtsheim (2009).

In much of the recent split-plot design literature, the equivalence of ordinary least squares (OLS) and GLS has received substantial attention. Letsinger et al. (1996) provided a proof of the equivalence of OLS and GLS estimators of the model parameters for crossed split-plot designs. Goos (2002) proved that, for saturated designs (a design for which the number of observations is equal to the number of model parameters), OLS and GLS are equivalent. Vining, Kowalski and Montgomery (2005) discussed the modification of central composite and Box-Behnken designs to accommodate a split-plot structure for a second-order response surface model. They also discussed some special cases of these designs where OLS and GLS estimators of the model parameters are equivalent and outlined some general conditions necessary for this property to be fulfilled. These designs are nowadays called equivalent-estimation split-plot designs. More recent work described strategies for constructing equivalent-estimation split-plot designs while at the same time giving the general equivalence condition of OLS and GLS for the split-plot 
design model. Parker, Kowalski and Vining (2006, 2007a) discussed two systematic design construction strategies to build balanced equivalent-estimation split-plot designs from modified BoxBehnken and central composite designs, while Parker, Kowalski and Vining (2007b) extended these techniques to accommodate unbalanced equivalent-estimation split-plot designs based on central-composite and Box-Behnken designs.

Goos (2006) compared the efficiency of D-optimal split-plot designs with that of equivalentestimation designs and reported various instances where the equivalent-estimation designs proposed in the literature were highly inefficient. At the same time, he discovered various D-optimal designs for which OLS and GLS are equivalent. Also, Parker, Kowalski and Vining (2007a) report a few instances involving one whole-plot factor where the D-optimal design is an equivalentestimation design.

A systematic study of the relationship between D-optimality and the equivalent-estimation property is an important void in the split-plot design literature. The purpose of this paper is to fill this void and to explore in detail when it is possible to find D-optimal designs for which OLS and GLS produce the same results. We focus on balanced split-plot designs, which have an equal number of subplots within every whole plot and which are the most practically relevant split-plot designs, and develop an algorithm that seeks the most efficient equivalent-estimation designs. First, however, we introduce the split-plot model, specify the condition for the equivalence of OLS and GLS, and define the D-optimality criterion. Next, we discuss several interesting designs produced by the algorithm and provide a catalog of all the scenarios in which we found D-efficient equivalent-estimation designs.

\section{The Split-Plot Design Model}

In this section, we introduce the linear model for split-plot designs. The general form of the splitplot design model for an experiment with $N$ runs, $b$ whole plots and $n=N / b$ runs or subplots per whole plot is given by

$$
\mathbf{y}=\mathbf{X} \boldsymbol{\beta}+\mathbf{Z} \gamma+\boldsymbol{\epsilon},
$$

where $\mathbf{y}$ is an $N \times 1$ vector of responses, $\mathbf{X}$ is an $N \times p$ design matrix containing the settings of the whole-plot factors, the subplot factors and their model expansions, $\boldsymbol{\beta}$ is a $p$-dimensional vector containing $p$ fixed effects in the model and $\mathbf{Z}$ is an $N \times b$ matrix of zeros and ones assigning the $N$ runs to the $b$ whole plots (i.e. the $(i, j)^{t h}$ element of $\mathbf{Z}$ is one if the $j^{\text {th }}$ run belongs to the $i^{\text {th }}$ whole plot and zero otherwise). The vector $\gamma$ is $b$-dimensional and contains the random effects of the $b$ whole plots. Finally, $\boldsymbol{\epsilon}$ is the $N$-dimensional vector of the random errors. It is assumed that $\gamma$ and $\boldsymbol{\epsilon}$ are uncorrelated, have mean zero and variances $\sigma_{\gamma}^{2} \mathbf{I}$ and $\sigma_{\epsilon}^{2} \mathbf{I}$ respectively. As a result, the assumed variance-covariance matrix of the model is given by

$$
\mathbf{V}=\sigma_{\epsilon}^{2} \mathbf{I}_{N}+\sigma_{\gamma}^{2} \mathbf{Z} \mathbf{Z}^{\prime}=\sigma_{\epsilon}^{2} \mathbf{I}_{N}+\sigma_{\gamma}^{2} \mathbf{D},
$$


where $\mathbf{I}_{N}$ is the identity matrix of size $N$ and

$$
\mathbf{D}=\left[\begin{array}{cccc}
\mathbf{1}_{n} \mathbf{1}_{n}^{\prime} & \mathbf{0}_{n} & \ldots & \mathbf{0}_{n} \\
\mathbf{0}_{n} & \mathbf{1}_{n} \mathbf{1}_{n}^{\prime} & \ldots & \mathbf{0}_{n} \\
\vdots & \vdots & \ddots & \vdots \\
\mathbf{0}_{n} & \mathbf{0}_{n} & \ldots & \mathbf{1}_{n} \mathbf{1}_{n}^{\prime}
\end{array}\right]
$$

where $\mathbf{0}_{n}$ is an $n \times n$ zero matrix. The covariance matrix $\mathbf{V}$ is block-diagonal, just like $\mathbf{D}$, which implies that observations in the same whole plot are correlated while those from different whole plots are not.

The GLS estimator of the factor effects is

$$
\hat{\boldsymbol{\beta}}_{G L S}=\left(\mathbf{X}^{\prime} \mathbf{V}^{-1} \mathbf{X}\right)^{-1} \mathbf{X}^{\prime} \mathbf{V}^{-1} \mathbf{y} .
$$

This estimator has the covariance matrix

$$
\operatorname{Var}\left(\hat{\boldsymbol{\beta}}_{G L S}\right)=\left(\mathbf{X}^{\prime} \mathbf{V}^{-1} \mathbf{X}\right)^{-1} \text {. }
$$

Though normally not recommended for this model given that it is less efficient than the GLS estimator, the OLS estimator is given by

$$
\hat{\boldsymbol{\beta}}_{O L S}=\left(\mathbf{X}^{\prime} \mathbf{X}\right)^{-1} \mathbf{X}^{\prime} \mathbf{y},
$$

which has a covariance matrix

$$
\operatorname{Var}\left(\hat{\boldsymbol{\beta}}_{O L S}\right)=\left(\mathbf{X}^{\prime} \mathbf{X}\right)^{-1} \mathbf{X}^{\prime} \mathbf{V X}\left(\mathbf{X}^{\prime} \mathbf{X}\right)^{-1}
$$

if the split-plot model is valid. For equivalent-estimation split-plot designs, the OLS and GLS estimators given above are the same, i.e.

$$
\hat{\boldsymbol{\beta}}_{O L S}=\hat{\boldsymbol{\beta}}_{G L S},
$$

in which case

$$
\operatorname{Var}\left(\hat{\boldsymbol{\beta}}_{O L S}\right)=\operatorname{Var}\left(\hat{\boldsymbol{\beta}}_{G L S}\right) .
$$

The equivalence of OLS and GLS is appealing because it implies that the $\mathbf{V}$ matrix and hence the variances $\sigma_{\gamma}^{2}$ and $\sigma_{\epsilon}^{2}$ need not be estimated in order to estimate the factor effects. This is especially important for researchers who do not have access to a software that allows restricted maximum likelihood estimation of variance components. However, it is worth noting that knowledge of the variance components remains essential for statistical inference of the estimated model.

\section{Split-Plot Design Construction Strategies}

As described by Goos (2006) and Jones and Nachtsheim (2009), there are several approaches for setting up split-plot response surface designs which have gained popularity in the literature. In this section we explore two of them: equivalent-estimation and optimal split-plot designs. First, we summarize the work that has been done on the construction of equivalent-estimation designs and point out what we believe is the major weakness of these designs. Next, we outline the optimal split-plot design approach. 


\subsection{Equivalent-Estimation Designs}

Equivalent-estimation split-plot designs have received considerable attention in the literature recently. They possess the property that the OLS estimator of the fixed effects in the splitplot model is equivalent to the GLS estimator. This property enables estimation of the fixed effects without estimating the variance components of the model. However, as reported by Goos (2006), many of the equivalent-estimation designs reported in the literature are lacking in terms of efficiency since they have a large number of replicated centre points.

The necessary and sufficient condition for equivalence of OLS and GLS estimates as given by McElroy (1967) is the existence of a $p \times p$ nonsingular matrix, $\mathbf{F}$, such that

$$
\mathbf{X F}=\mathbf{V X}
$$

Parker et al. (2007a) give a general form of the equivalence condition tailored to split-plot designs. By substituting Equation (2) in Equation (3) and pre-multiplying by $\left(\mathbf{X}^{\prime} \mathbf{X}\right)^{-1} \mathbf{X}^{\prime}$ they find that

$$
\mathbf{F}=\sigma_{\epsilon}^{2} \mathbf{I}+\sigma_{\gamma}^{2} \mathbf{K}
$$

where $\mathbf{K}=\left(\mathbf{X}^{\prime} \mathbf{X}\right)^{-1} \mathbf{X}^{\prime} \mathbf{D X}$. Combining Equations (3) and (4) and simplifying then leads to the following condition for the equivalence of OLS and GLS:

$$
\mathbf{X K}=\mathbf{D X}
$$

It is this expression which we use in our modified coordinate-exchange algorithm to numerically check the equivalence of OLS and GLS when constructing D-efficient equivalent-estimation designs.

\subsection{Optimal Split-Plot Designs}

D-optimality is the most commonly used optimality criterion for selecting experimental designs. This criterion seeks to minimize the generalized variance of the parameter estimates, which is done by minimizing the determinant of the variance-covariance matrix of the factor effects' estimates or equivalently, by maximizing the determinant of the information matrix about $\boldsymbol{\beta}$. For a split-plot design, the information matrix is given by

$$
\mathbf{M}=\mathbf{X}^{\prime} \mathbf{V}^{-1} \mathbf{X}
$$

when the GLS estimator is used.

The efficiency of a design is obtained by comparing the determinant of its information matrix with that of the corresponding D-optimal design. As shown in Goos (2002), D-optimal designs depend on the relative magnitude of $\sigma_{\gamma}^{2}$ and $\sigma_{\epsilon}^{2}$, but not on their absolute magnitude. Letting $\mathbf{M}$ be the information matrix of the D-optimal design with design matrix $\mathbf{X}$ and $\mathbf{M}_{\mathbf{A}}$ be the 
information matrix of a design with design matrix $\mathbf{A}$ for the same design problem, the relative D-efficiency of the design corresponding to $\mathbf{A}$ is defined as

$$
\mathbf{D}_{\text {eff }}=\left\{\frac{\left|\mathbf{M}_{\mathbf{A}}\right|}{|\mathbf{M}|}\right\}^{1 / p}=\left\{\frac{\left|\mathbf{A}^{\prime} \mathbf{V}^{-1} \mathbf{A}\right|}{\left|\mathbf{X}^{\prime} \mathbf{V}^{-1} \mathbf{X}\right|}\right\}^{1 / p},
$$

where $p$ is the number of parameters in the model. This relative efficiency provides the percentage amount of information contained in a design compared to the D-optimal design.

Goos and Vandebroek (2001, 2003, 2004) developed point-exchange algorithms for constructing split-plot designs that require specification of a candidate-set containing all the possible allowable combinations of the factor levels (i.e. all possible design points). The point-exchange algorithms start from an initial design, that is partly generated at random and partly in a greedy fashion. They then proceed by exchanging designs points from the initial design with points from the candidate set until D-optimality is obtained. The construction of a candidate set can be problematic when the number of experimental factors is large and/or the experimental space is highly constrained. To avoid this problem, Jones and Goos (2007) described a flexible candidate-setfree coordinate-exchange algorithm for constructing D-optimal split-plot designs. The algorithm starts from an initial design generated randomly and then tries to improve this design coordinate by coordinate for a prespecified number of times maximizing the D-optimality criterion.

\section{Constructing D-efficient Equivalent-Estimation Designs}

In this section, we outline an iterative algorithm to construct balanced D-efficient equivalentestimation designs. The algorithm is a modification of the candidate-set-free coordinate-exchange algorithm of Jones and Goos (2007). The modified algorithm consists of two parts. First, a starting design is generated randomly. Next, the algorithm proceeds with a coordinate-by-coordinate improvement of the starting design. As local search optimization methods such as coordinateexchange algorithms are prone to getting stuck in a local optimum, we run our coordinateexchange algorithm $T$ times, each time starting from a different random initial design. This is common practice in optimal experimental design.

Below, we describe the input and the output of the algorithm, and sketch its two main parts. A step-by-step description of the algorithm is given in Algorithm 1. In the step-by-step description, the $\mathrm{D}$-criterion value of the current design is denoted by $D_{c}$. The current best $\mathrm{D}$-criterion value found for a given random start is denoted by $D$, while the best $\mathrm{D}$-criterion value found over all random starts of the algorithm is denoted by $D_{\text {opt }}$. The D-criterion value for the current best equivalence-estimation design for a given random start is denoted by $D_{e q}$, while the overall best D-criterion value for an equivalent-estimation design is denoted by $D_{\text {opteq }}$. 


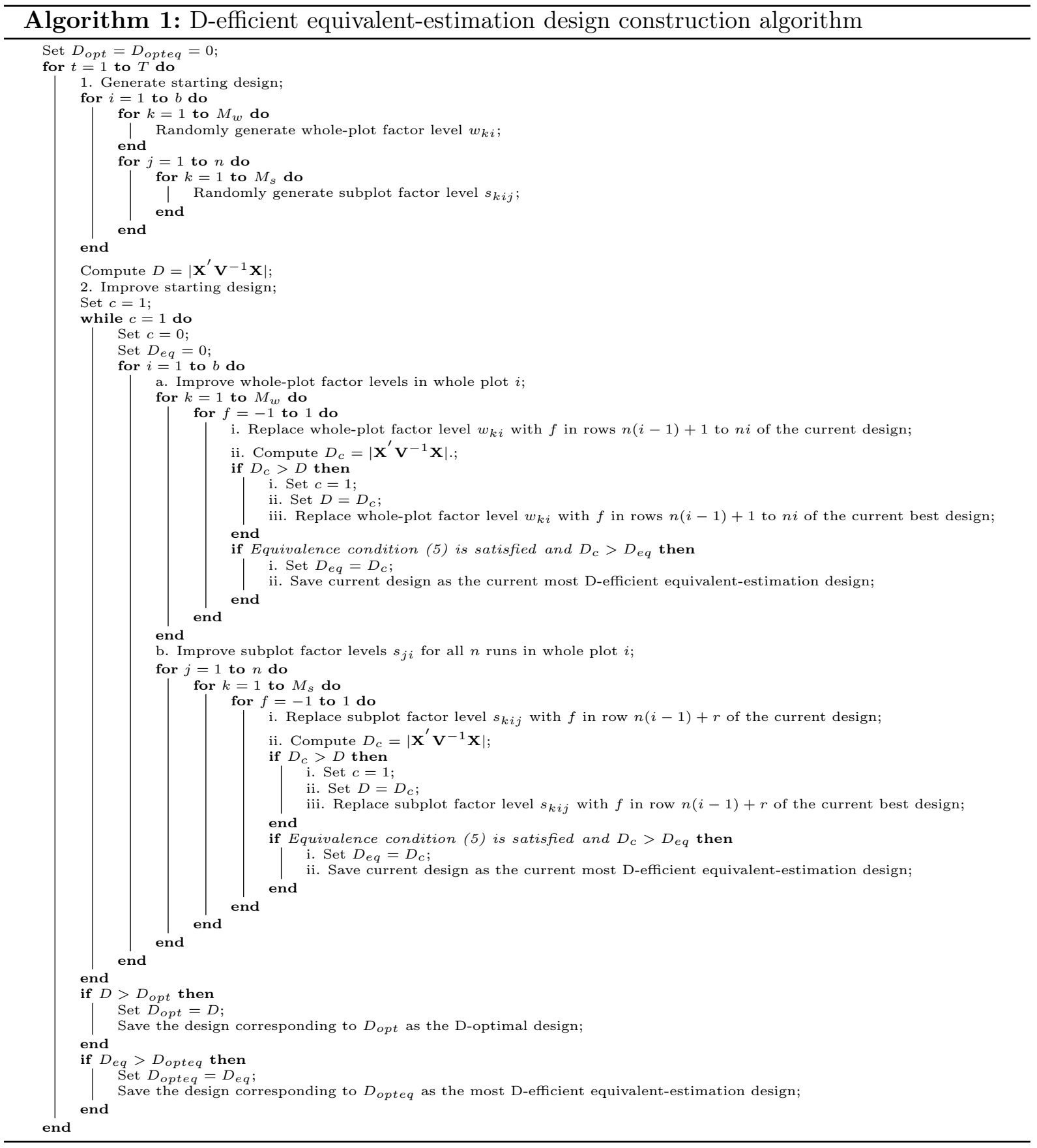




\subsection{Input to the Algorithm}

The algorithm requires the following inputs:

- the sample size, $N$;

- the number of whole plots, $b$;

- the number of whole-plot factors, $M_{w}$;

- the number of subplot factors, $M_{s}$;

- the ratio of the whole-plot error variance to the subplot error variance, $\eta=\sigma_{\gamma}^{2} / \sigma_{\epsilon}^{2}$.

When these input parameters are known, the $\mathbf{D}$ matrix, required to check the OLS-GLS equivalence using Equation (5), can be constructed. The whole-plot size $n$ can also be obtained from $N$ and $b: n=N / b$. The algorithm we developed assumes that the fitted model is a second-order response surface model involving an intercept, $M_{w}+M_{s}$ main effects, $M_{w}+M_{s}$ quadratic effects and $\left(M_{w}+M_{s}\right)\left(M_{w}+M_{s}-1\right) / 2$ two-factor interactions. The algorithm therefore generates three-level designs. For the full second-order model, the $j^{\text {th }}$ observation in whole plot $i$ is given by

$$
\begin{array}{r}
y_{i j}=\beta_{0}+\sum_{k=1}^{M_{w}}\left(\beta_{k}^{w} w_{k i}+\beta_{k k}^{w} w_{k i}^{2}\right)+\sum_{k=1}^{M_{s}}\left(\beta_{k}^{s} s_{k i j}+\beta_{k k}^{s} s_{k i j}^{2}\right)+\sum_{k=1}^{M_{w}} \sum_{l=k+1}^{M_{w}} \beta_{k l}^{w w} w_{k i} w_{l i} \\
+\sum_{k=1}^{M_{s}} \sum_{l=k+1}^{M_{s}} \beta_{k l}^{s s} s_{k i j} s_{l i j}+\sum_{k=1}^{M_{w}} \sum_{l=1}^{M_{s}} \beta_{k l}^{w s} w_{k i} s_{l i j}+\gamma_{i}+\epsilon_{i j},
\end{array}
$$

where $w_{k i}$ represents the level of the $k^{t h}$ whole-plot factor in whole plot $i, s_{k i j}$ represents the level of the $k^{t h}$ subplot factor at the $j^{\text {th }}$ run in whole plot $i, \gamma_{i}$ is the random effect of whole plot $i$, and $\epsilon_{i j}$ is the random error for the $j^{\text {th }}$ run in whole plot $i$.

As mentioned earlier, the construction of equivalent-estimation design does not require knowledge of the variance components. However, the construction of D-optimal designs and D-efficient equivalent-estimation designs depends on the whole-plot error variance and the sub-plot error variance through $\eta$. As recommended by Goos (2002), who pointed out that D-optimal designs and relative D-efficiencies are relatively insensitive to $\eta$, we have used an $\eta$ value of one in our computations. For this reason, the relative D-efficiencies we report below were all computed assuming $\eta=1$.

\subsection{Output of the Algorithm}

Usually the algorithm generates two outputs. The first output is the D-optimal design with its corresponding D-criterion value. The second output is the most D-efficient equivalent-estimation 
design and its D-criterion value. This allows us to see the cost, in terms of D-efficiency, of using an equivalent-estimation design. As we shall see below, in some cases, the two designs produced by the algorithm are identical. Generally, however, the most D-efficient equivalent-estimation design is slightly less efficient than the D-optimal design. In some cases, the algorithm did not find any equivalent-estimation design. In that case, the algorithm's output only contains the D-optimal design.

\subsection{Generating a Starting Design}

The starting design is randomly generated column-by-column. All the required factor levels of the starting design are generated from the uniform distribution over the interval $[-1,1]$. The layout of the design is in such a way that the whole-plot factors are listed in the leftmost columns while the subplot factors are in the rightmost columns. Therefore, $b$ random levels are generated first for each whole-plot factor. Next, $n$ random levels are generated for each of the subplot factors. The construction is in such a way that the initial design obeys the desired split-plot structure where the whole-plot factor levels vary from whole plot to whole plot, while the subplot factor levels vary from subplot to subplot. Obviously, at this point, it is unlikely to find a random starting design for which the OLS and GLS estimators are equal. Hence, only the D-criterion value of this design is computed at this stage of the algorithm.

\subsection{Improving the Starting Design}

For each random start of the algorithm, the starting design is improved iteratively from whole plot to whole plot and from one run to the other. To this end, each factor level in the initial design, i.e. each coordinate of the $N$ design points, is exchanged with $-1,0$ or 1 . An exchange of whole-plot factor levels is different from that of subplot factor levels since a change to the level of a whole-plot factor in a given whole plot induces a similar change to the other levels of that factor in the same whole plot. At each exchange, the D-criterion value of the resulting design is computed. If a level results in a higher value of the D-criterion value, it is retained in the current design. Otherwise no changes are made to the design. At the same time, on every exchange, the equivalence condition given by Equation (5) is also evaluated. If the design satisfies this condition, its D-criterion value is compared to that of the best previously found equivalent-estimation design. If the $\mathrm{D}$-criterion value of the newly found equivalent-estimation design is the better of the two, it is saved. The coordinate exchanges continue until no further improvements can be made to the design in terms of the $\mathrm{D}$-criterion value.

This procedure is repeated for each of the $T$ random starts of the algorithm, and the overall best design in terms of the D-optimality criterion and the best equivalent-estimation design in terms of the D-optimality criterion are provided as the output.

The main difference between our algorithm and the candidate-set-free algorithm of Jones and Goos (2007) is that, for every intermediate design produced, our algorithm checks whether it 
Table 1: A D-optimal and a most D-efficient equivalent-estimation design with one whole-plot factor $w$, one subplot factor $s$, four whole plots and eight runs

\begin{tabular}{crrrrr}
\hline \multirow{2}{*}{ Whole plot } & \multicolumn{2}{c}{ D-optimal } & & \multicolumn{2}{c}{ Eqv-Estim } \\
\cline { 2 - 3 } \cline { 5 - 6 } & $w$ & $s$ & & $w$ & $s$ \\
\hline 1 & -1 & -1 & & -1 & -1 \\
& -1 & 1 & & -1 & 1 \\
\hline 2 & -1 & 1 & & 0 & -1 \\
& -1 & 0 & & 0 & 0 \\
\hline 3 & 0 & -1 & & 0 & -1 \\
& 0 & 0 & 0 & 0 \\
\hline \multirow{2}{*}{4} & 1 & -1 & 1 & -1 \\
& 1 & 1 & 1 & 1 \\
\hline
\end{tabular}

is an equivalent-estimation design. If so, its D-criterion value is compared to that of the best current equivalent-estimation design.

\section{$5 \quad$ Illustrations}

In this section, we discuss in detail some of the results that we obtained from our algorithm. The cases we selected to discuss here range from expected to completely surprising, and from problems involving as few as two experimental factors up to problems involving as many as six factors.

\subsection{Two small examples}

Table 1 shows two designs with one whole-plot factor $w$, one subplot factor $s$ and four whole plots with two subplots each. The left panel shows the D-optimal design while the right panel shows the corresponding most D-efficient equivalent-estimation design.

The D-efficiency of the equivalent-estimation design relative to the D-optimal design is $94 \%$ and therefore the loss of information when we use this design instead of the D-optimal design is small. An important feature of this design is that, in spite of its small size, it allows for the estimation of all the parameters associated with the hard-to-change variable in a second-order model, including the whole-plot error variance $\sigma_{\gamma}^{2}$. This is because there are four whole-plot degrees of freedom which are used for estimating the intercept, the whole-plot main effect, the whole-plot quadratic effect and the whole-plot error variance. The simplicity of this design allows 
Table 2: D-optimal design with one whole-plot factor $w$, one subplot factor $s$, five whole plots and 15 runs for which the OLS and GLS estimators are equivalent

\begin{tabular}{crr}
\hline Whole plot & $w$ & $s$ \\
\hline 1 & -1 & -1 \\
& -1 & 0 \\
& -1 & 1 \\
\hline 2 & -1 & -1 \\
& -1 & 0 \\
& -1 & 1 \\
\hline 3 & 0 & -1 \\
& 0 & 0 \\
& 0 & 1 \\
\hline 4 & 1 & -1 \\
& 1 & 0 \\
& 1 & 1 \\
\hline 5 & 1 & -1 \\
& 1 & 0 \\
& 1 & 1 \\
\hline
\end{tabular}

us to manually verify the equivalence condition as given in Equation (3).

While orthogonality is a desirable property in general, our first example shows that this is not a necessary condition for the equivalence of OLS and GLS. Indeed, the levels of the subplot factor $s$ in the equivalent-estimation design in Table 1 are not even balanced across the complete design, let alone within whole plots. This makes the equivalent-estimation design in Table 1 completely different from the designs in Parker et al. (2006, 2007a, 2007b) which are level-balanced by construction, and have a subplot design that is orthogonal to the whole plots.

While our modified coordinate exchange algorithm does not impose orthogonality, it occasionally returns a D-optimal design in which the design for the subplot factors is orthogonal to the whole plots. In the D-optimal 15-run design with five whole plots for one whole-plot factor and one subplot factor shown in Table 2, the subplot main effect and whole-plot to subplot factor interaction sum to zero in every whole plot indicating orthogonality. Because the same levels of the subplot factors are used in every whole plot, the design in Table 2 is crossed. Letsinger et al. (1996) showed that crossed split-plot designs are equivalent-estimation designs. This is one of the few cases we found where the most D-efficient equivalent-estimation design is actually the D-optimal design, i.e. the D-efficiency of the final equivalent-estimation design was $100 \%$. 


\subsection{Examples with more than two factors}

One extension to the design in Table 2 would be to add more subplot factors. Even adding only one extra subplot factor results in the loss of orthogonality and OLS-GLS equivalence for the D-optimal design. This can be seen in Table 3, where we show the D-optimal design and the most D-efficient equivalent-estimation design with one whole-plot factor $w$, two subplot factors $s_{1}$ and $s_{2}$, and five whole plots of three runs each. The most D-efficient equivalent-estimation design has a D-efficiency of $92 \%$. In general, adding factors to an already equivalent-estimation design destroys the equivalence of OLS and GLS.

Table 3: A D-optimal and a most D-efficient equivalent-estimation design with one whole-plot factor $w$, two subplot factors $s_{1}$ and $s_{2}$, five whole plots and 15 runs

\begin{tabular}{crrrrrrrr}
\hline & \multicolumn{3}{c}{ D-optimal } & & \multicolumn{3}{c}{ Eqv-Estim } \\
\cline { 2 - 5 } \cline { 6 - 8 } Whole plot & $w$ & $s_{1}$ & $s_{2}$ & & $w$ & $s_{1}$ & $s_{2}$ \\
\hline 1 & -1 & -1 & 0 & & -1 & -1 & 0 \\
& -1 & 0 & 1 & & -1 & 0 & 1 \\
& -1 & 1 & -1 & & -1 & 1 & -1 \\
\hline 2 & -1 & -1 & 1 & & -1 & -1 & 1 \\
& -1 & -1 & -1 & & -1 & 0 & -1 \\
& -1 & 1 & 0 & & -1 & 1 & 0 \\
\hline 3 & 0 & -1 & 1 & & 0 & -1 & -1 \\
& 0 & 0 & 0 & & 0 & 0 & 0 \\
& 0 & 1 & 1 & & 0 & 1 & -1 \\
\hline \multirow{2}{*}{4} & 1 & -1 & -1 & & 1 & -1 & -1 \\
& 1 & 0 & 1 & & 1 & 0 & 1 \\
& 1 & 1 & -1 & & 1 & 1 & 0 \\
\hline 5 & 1 & -1 & 1 & & 1 & -1 & 0 \\
& 1 & 0 & -1 & & 1 & 0 & -1 \\
& 1 & 1 & 1 & & 1 & 1 & 1 \\
\hline
\end{tabular}

In practice, one is often faced with more than one factor whose levels cannot be changed easily. Our modified coordinate-exchange algorithm is able to find equivalent-estimation designs for these cases as well. Table 4 shows a D-optimal design and the most D-efficient equivalentestimation design with two whole-plot factors $w_{1}$ and $w_{2}$, one subplot factor $s$ and seven whole plots with two subplots each. Surprisingly, with such values as 14 runs and seven whole plots, it is still possible to get an efficient equivalent-estimation design. We find it remarkable that it is possible to find a three-level design with two subplots per whole-plot for which the OLS and GLS estimators are equivalent. It turns out that the D-efficiency of that equivalent-estimation design is $94 \%$. 
Table 4: A D-optimal and a most D-efficient equivalent-estimation design with two whole-plot factors $w_{1}$ and $w_{2}$, one subplot factor $s$, seven whole plots and 14 runs

\begin{tabular}{crrrrrrrr}
\hline & \multicolumn{3}{c}{ D-optimal } & & \multicolumn{3}{c}{ Eqv-Estim } \\
\cline { 2 - 4 } \cline { 6 - 8 } Whole plot & $w_{1}$ & $w_{2}$ & $s$ & & $w_{1}$ & $w_{2}$ & $s$ \\
\hline 1 & -1 & -1 & -1 & & -1 & -1 & -1 \\
& -1 & -1 & 1 & & -1 & -1 & 1 \\
\hline 2 & -1 & 1 & -1 & & -1 & 1 & -1 \\
& -1 & 1 & 1 & & -1 & 1 & 1 \\
\hline 3 & 0 & -1 & 0 & & -1 & 0 & 0 \\
& 0 & -1 & 1 & & -1 & 0 & 1 \\
\hline \multirow{2}{*}{4} & 0 & 0 & -1 & & 0 & 1 & -1 \\
& 0 & 0 & 0 & & 0 & 1 & 0 \\
\hline 5 & 1 & -1 & -1 & & 1 & -1 & -1 \\
& 1 & -1 & 1 & & -1 & 1 \\
\hline \multirow{2}{*}{6} & 1 & 0 & 0 & & 1 & -1 & -1 \\
& 1 & 0 & 1 & & 1 & -1 & 1 \\
\hline 7 & 1 & 1 & -1 & & 1 & 1 & -1 \\
& 1 & 1 & 1 & 1 & 1 & 1 \\
\hline
\end{tabular}

\subsection{Two examples with multiple whole-plot and subplot factors}

An even more complex equivalent-estimation design is given in Table 5 along with the corresponding D-optimal design. This design has three whole-plot factors $w_{1}, w_{2}$ and $w_{3}$, three subplot factors $s_{1}, s_{2}$ and $s_{3}$, twelve whole plots and four runs per whole plot. This design illustrates that it is still possible to generate good and complex equivalent-estimation designs for larger numbers of factors. In this case, the most D-efficient equivalent-estimation design has a D-efficiency of $93 \%$.

While the design given in Table 2 is D-optimal, equivalent-estimation as well as orthogonal at the subplot level, it is worth noting that orthogonality at the subplot level is not necessary. This is clearly illustrated by the design shown on Table 6 . This design with three whole-plot factors $w_{1}, w_{2}$ and $w_{3}$, two subplot factors $s_{1}$ and $s_{2}$ and ten whole plots with three runs each. It is both D-optimal and equivalent estimation. However, the subplot factor levels in each whole plot do not sum to zero and, hence, the design is not orthogonal. An interesting aspect of this example is that it demonstrates that the equivalence of OLS and GLS can also be achieved for large designs involving many factors. Thus, the simultaneous appearance of the features 'D-optimality' and 'OLS-GLS equivalence' is not restricted to small designs that accommodate only a few factors. 
Table 5: A D-optimal and a most D-efficient equivalent-estimation design with three whole-plot factors $w_{1}, w_{2}$ and $w_{3}$, three subplot factors $s_{1}, s_{2}$ and $s_{3}, 12$ whole plots and 48 runs

\begin{tabular}{|c|c|c|c|c|c|c|c|c|c|c|c|c|}
\hline \multirow[b]{2}{*}{ Whole plot } & \multicolumn{6}{|c|}{ D-optimal } & \multicolumn{6}{|c|}{ Eqv-Estim } \\
\hline & $w_{1}$ & $w_{2}$ & $w_{3}$ & $s_{1}$ & $s_{2}$ & $s_{3}$ & $w_{1}$ & $w_{2}$ & $w_{3}$ & $s_{1}$ & $s_{2}$ & $s_{3}$ \\
\hline \multirow[t]{4}{*}{1} & -1 & -1 & -1 & -1 & 1 & 0 & -1 & -1 & -1 & -1 & 0 & -1 \\
\hline & -1 & -1 & -1 & 0 & -1 & -1 & -1 & -1 & -1 & 0 & -1 & 1 \\
\hline & -1 & -1 & -1 & 1 & 0 & 1 & -1 & -1 & -1 & 1 & -1 & -1 \\
\hline & -1 & -1 & -1 & 1 & 1 & -1 & -1 & -1 & -1 & 1 & 1 & 1 \\
\hline \multirow[t]{4}{*}{2} & -1 & -1 & 1 & -1 & -1 & 1 & -1 & -1 & 1 & -1 & -1 & -1 \\
\hline & -1 & -1 & 1 & -1 & 1 & -1 & -1 & -1 & 1 & -1 & 1 & 1 \\
\hline & -1 & -1 & 1 & 1 & -1 & -1 & -1 & -1 & 1 & 1 & -1 & 1 \\
\hline & -1 & -1 & 1 & 1 & 1 & 1 & -1 & -1 & 1 & 1 & 1 & -1 \\
\hline \multirow[t]{4}{*}{3} & -1 & 1 & -1 & -1 & -1 & -1 & -1 & 1 & -1 & -1 & -1 & -1 \\
\hline & -1 & 1 & -1 & -1 & 1 & 1 & -1 & 1 & -1 & -1 & 1 & 1 \\
\hline & -1 & 1 & -1 & 1 & -1 & 1 & -1 & 1 & -1 & 1 & -1 & 1 \\
\hline & -1 & 1 & -1 & 1 & 1 & -1 & -1 & 1 & -1 & 1 & 1 & -1 \\
\hline \multirow[t]{4}{*}{4} & -1 & 0 & 0 & -1 & -1 & 0 & -1 & 1 & -1 & -1 & -1 & 1 \\
\hline & -1 & 0 & 0 & 0 & 1 & 1 & -1 & 1 & -1 & -1 & 1 & -1 \\
\hline & -1 & 0 & 0 & 1 & -1 & 1 & -1 & 1 & -1 & 1 & -1 & -1 \\
\hline & -1 & 0 & 0 & 1 & 0 & -1 & -1 & 1 & -1 & 1 & 1 & 1 \\
\hline \multirow[t]{4}{*}{5} & -1 & 1 & 1 & 1 & -1 & -1 & -1 & 0 & 0 & 0 & 0 & 0 \\
\hline & -1 & 1 & 1 & -1 & 1 & -1 & -1 & 0 & 0 & -1 & -1 & 1 \\
\hline & -1 & 1 & 1 & 1 & 1 & 1 & -1 & 0 & 0 & -1 & 1 & -1 \\
\hline & -1 & 1 & 1 & -1 & -1 & 1 & -1 & 0 & 0 & 1 & -1 & -1 \\
\hline \multirow[t]{4}{*}{6} & 0 & -1 & 0 & -1 & 0 & -1 & 0 & -1 & 0 & -1 & -1 & 1 \\
\hline & 0 & -1 & 0 & -1 & 1 & 1 & 0 & -1 & 0 & 0 & 1 & 0 \\
\hline & 0 & -1 & 0 & 0 & -1 & 1 & 0 & -1 & 0 & 1 & -1 & -1 \\
\hline & 0 & -1 & 0 & 1 & 1 & 0 & 0 & -1 & 0 & 1 & 0 & 1 \\
\hline \multirow[t]{4}{*}{7} & 0 & 0 & -1 & -1 & -1 & -1 & 0 & 0 & 1 & -1 & -1 & -1 \\
\hline & 0 & 0 & -1 & -1 & 0 & 1 & 0 & 0 & 1 & -1 & 1 & 0 \\
\hline & 0 & 0 & -1 & 0 & 1 & -1 & 0 & 0 & 1 & 0 & 0 & 1 \\
\hline & 0 & 0 & -1 & 1 & -1 & 0 & 0 & 0 & 1 & 1 & 1 & -1 \\
\hline \multirow[t]{4}{*}{8} & 1 & -1 & -1 & -1 & -1 & 1 & 1 & -1 & -1 & -1 & -1 & -1 \\
\hline & 1 & -1 & -1 & -1 & 1 & -1 & 1 & -1 & -1 & -1 & 1 & 1 \\
\hline & 1 & -1 & -1 & 1 & -1 & -1 & 1 & -1 & -1 & 1 & -1 & 1 \\
\hline & 1 & -1 & -1 & 1 & 1 & 1 & 1 & -1 & -1 & 1 & 1 & -1 \\
\hline \multirow[t]{4}{*}{9} & 1 & -1 & 1 & -1 & -1 & -1 & 1 & -1 & 1 & -1 & -1 & 1 \\
\hline & 1 & -1 & 1 & -1 & 1 & 1 & 1 & -1 & 1 & -1 & 1 & -1 \\
\hline & 1 & -1 & 1 & 1 & -1 & 1 & 1 & -1 & 1 & 1 & -1 & 0 \\
\hline & 1 & -1 & 1 & 1 & 1 & -1 & 1 & -1 & 1 & 1 & 1 & 1 \\
\hline \multirow{4}{*}{10} & 1 & 1 & -1 & -1 & -1 & 1 & 1 & 1 & 1 & -1 & 0 & 1 \\
\hline & 1 & 1 & -1 & -1 & 1 & 0 & 1 & 1 & 1 & 0 & -1 & -1 \\
\hline & 1 & 1 & -1 & 1 & 0 & -1 & 1 & 1 & 1 & 1 & -1 & 1 \\
\hline & 1 & 1 & -1 & 1 & 1 & 1 & 1 & 1 & 1 & 1 & 1 & 0 \\
\hline \multirow[t]{4}{*}{11} & 1 & 1 & 0 & -1 & 1 & -1 & 1 & 1 & -1 & -1 & -1 & 1 \\
\hline & 1 & 1 & 0 & 0 & 0 & 0 & 1 & 1 & -1 & -1 & 1 & -1 \\
\hline & 1 & 1 & 0 & 1 & -1 & -1 & 1 & 1 & -1 & 1 & -1 & -1 \\
\hline & 1 & 1 & 0 & 1 & 1 & 1 & 1 & 1 & -1 & 1 & 1 & 1 \\
\hline \multirow[t]{4}{*}{12} & 1 & 1 & 1 & -1 & -1 & -1 & 1 & 1 & 1 & -1 & -1 & 0 \\
\hline & 1 & 1 & 1 & -1 & 1 & 1 & 1 & 1 & 1 & 0 & 1 & 1 \\
\hline & 1 & 1 & 1 & 1 & -1 & 1 & 1 & 1 & 1 & 1 & -1 & 1 \\
\hline & 1 & 1 & 1 & 1 & 1 & -1 & 1 & 1 & 1 & 1 & 0 & -1 \\
\hline
\end{tabular}


Table 6: A D-optimal design with three whole-plot factors $w_{1}, w_{2}$ and $w_{3}$, two subplot factors $s_{1}$ and $s_{2}$, ten whole plots and 30 runs for which the OLS and GLS estimators are equivalent

\begin{tabular}{|c|c|c|c|c|c|}
\hline Whole plot & $w_{1}$ & $w_{2}$ & $w_{3}$ & $s_{1}$ & $s_{2}$ \\
\hline \multirow[t]{3}{*}{1} & -1 & -1 & -1 & -1 & 0 \\
\hline & -1 & -1 & -1 & 1 & -1 \\
\hline & -1 & -1 & -1 & 1 & 1 \\
\hline \multirow[t]{3}{*}{2} & -1 & -1 & 1 & -1 & -1 \\
\hline & -1 & -1 & 1 & 0 & 1 \\
\hline & -1 & -1 & 1 & 1 & -1 \\
\hline \multirow[t]{3}{*}{3} & -1 & 1 & -1 & -1 & 1 \\
\hline & -1 & 1 & -1 & 0 & -1 \\
\hline & -1 & 1 & -1 & 1 & 1 \\
\hline \multirow[t]{3}{*}{4} & -1 & 0 & 0 & -1 & -1 \\
\hline & -1 & 0 & 0 & -1 & 1 \\
\hline & -1 & 0 & 0 & 1 & 0 \\
\hline \multirow[t]{3}{*}{5} & 0 & 0 & 1 & -1 & 1 \\
\hline & 0 & 0 & 1 & 0 & -1 \\
\hline & 0 & 0 & 1 & 1 & 0 \\
\hline \multirow[t]{3}{*}{6} & 0 & 1 & 0 & -1 & -1 \\
\hline & 0 & 1 & 0 & 0 & 1 \\
\hline & 0 & 1 & 0 & 1 & 0 \\
\hline \multirow[t]{3}{*}{7} & 1 & -1 & -1 & -1 & -1 \\
\hline & 1 & -1 & -1 & -1 & 1 \\
\hline & 1 & -1 & -1 & 1 & 0 \\
\hline \multirow[t]{3}{*}{8} & 1 & -1 & 1 & -1 & 1 \\
\hline & 1 & -1 & 1 & 0 & -1 \\
\hline & 1 & -1 & 1 & 1 & 1 \\
\hline \multirow[t]{3}{*}{9} & 1 & 1 & -1 & -1 & -1 \\
\hline & 1 & 1 & -1 & 0 & 1 \\
\hline & 1 & 1 & -1 & 1 & -1 \\
\hline \multirow[t]{3}{*}{10} & 1 & 1 & 1 & -1 & 0 \\
\hline & 1 & 1 & 1 & 1 & -1 \\
\hline & 1 & 1 & 1 & 1 & 1 \\
\hline
\end{tabular}




\subsection{A catalog of equivalent-estimation designs}

We have run our modified coordinate-exchange algorithm with 1000 tries for a broad range of input settings. We attempted to generate efficient equivalent-estimation designs with up to three whole-plot factors and up to three subplot factors, and studied whole-plot sizes between two and six. A summary of our results is given in Table 7. The table shows that it is possible to find highly efficient equivalent-estimation designs in many cases. The worst D-efficiency we obtained for an equivalent-estimation design is $87.9 \%$. Files containing the designs reported in Table 7 are available from the authors.

\section{Discussion}

Split-plot designs are very effective in reducing the cost of an experiment in the presence of hardto-change factors. In general, the bi-randomization structure of the split-plot design requires the use of generalized least squares (GLS) to estimate the model. This estimation approach is not very straightforward and may not be implemented in the statistical software available to a practitioner. This has led to the development of various methods for constructing split-plot designs for which the OLS and GLS estimators produce the same point estimates. Often, these designs were provided statistically inefficient estimates of the fixed effects. In this paper we have outlined an algorithm for constructing D-efficient equivalent-estimation split-plot designs. We have shown that it is possible to obtain highly D-efficient equivalent-estimation split-plot designs such that the loss of precision in parameter estimates is negligible if OLS is the preferred estimation technique. We have also shown that adding more factors to an already equivalentestimation design may destroy the equivalence property.

An interesting fact is that whether or not a design is an equivalent-estimation design depends on the model actually fitted. The equivalent-estimation designs that we listed in this article all possess the property that the OLS and GLS estimators are equivalent if the full second-order response surface model is estimated. Moreover, dropping subplot quadratic effects as well as the interactions associated with the subplot factors does not destroy the equivalence property. However, dropping any of the terms associated with the whole-plot factors destroys the OLS-GLS equivalence. Thus, reducing the model complexity leads to the loss of the OLS-GLS equivalence property. This is counterintuitive, as desirable theoretical properties are usually easier to achieve for simple models. In any case, the fact that the OLS-GLS property is model dependent implies that equivalent-estimation design should be used with care because the OLS estimator might no longer be as efficient as the GLS estimator if the model is simplified.

We have provided a broad range of new equivalent-estimation designs, each of which is highly efficient. Moreover, our modified coordinate-exchange algorithm allows the construction of Defficient equivalent-estimation designs for models other than the second-order models we studied here. An interesting avenue for future research would be to investigate the existence of equivalent-estimation split-split-plot or strip-plot designs. While Jones and Goos (2009) report 
Table 7: A summary of the cases for which we tried to find equivalent-estimation designs, along with the D-efficiency of the most efficient equivalent-estimation designs we obtained

\begin{tabular}{|c|c|c|c|c|c|c|c|c|c|c|c|c|c|c|}
\hline$M_{w}$ & $M_{s}$ & $b$ & $n$ & D-eff & $M_{w}$ & $M_{s}$ & $b$ & $n$ & D-eff & $M_{w}$ & $M_{s}$ & $b$ & $n$ & D-eff \\
\hline \multirow[t]{50}{*}{1} & 1 & 3 & 2 & 1 & & 3 & 3 & 2 & - & & & 12 & 2 & - \\
\hline & & & 3 & 1 & & & & 3 & - & & & & 3 & 0.998 \\
\hline & & & 4 & 1 & & & & 4 & - & & & & 4 & - \\
\hline & & & 5 & 1 & & & & 5 & 1 & & & & 5 & 0.999 \\
\hline & & & 6 & 1 & & & & 6 & 1 & & & & 6 & - \\
\hline & & 4 & 2 & 0.933 & & & 4 & 2 & - & & 2 & 6 & 2 & 1 \\
\hline & & & 3 & 1 & & & & 3 & - & & & & 3 & 1 \\
\hline & & & 4 & 0.994 & & & & 4 & 0.929 & & & & 4 & 1 \\
\hline & & & 5 & 1 & & & & 5 & 0.988 & & & & 5 & 1 \\
\hline & & & 6 & 1 & & & & 6 & 0.986 & & & & 6 & 1 \\
\hline & & 5 & 2 & 0.934 & & & 5 & 2 & - & & & 7 & 2 & - \\
\hline & & & 3 & 1 & & & & 3 & 1 & & & & 3 & 0.972 \\
\hline & & & 4 & 0.989 & & & & 4 & 0.966 & & & & 4 & 0.987 \\
\hline & & & 5 & 1 & & & & 5 & 0.989 & & & & 5 & 0.999 \\
\hline & & & 6 & 0.998 & & & & 6 & - & & & & 6 & 0.995 \\
\hline & & 6 & 2 & 0.971 & & & 6 & 2 & - & & & 8 & 2 & 0.965 \\
\hline & & & 3 & 1 & & & & 3 & 0.889 & & & & 3 & 0.913 \\
\hline & & & 4 & 0.991 & & & & 4 & - & & & & 4 & 0.970 \\
\hline & & & 5 & 0.999 & & & & 5 & - & & & & 5 & 0.999 \\
\hline & & & 6 & 0.998 & & & & 6 & - & & & & 6 & 0.962 \\
\hline & & 7 & 2 & 0.940 & 2 & 1 & 6 & 2 & 1 & & & 9 & 2 & 0.931 \\
\hline & & & 3 & 1 & & & & 3 & 1 & & & & 3 & - \\
\hline & & & 4 & 0.992 & & & & 4 & 1 & & & & 4 & 0.894 \\
\hline & & & 5 & 0.999 & & & & 5 & 1 & & & & 5 & 0.998 \\
\hline & & & 6 & 0.999 & & & & 6 & 1 & & & & 6 & 0.965 \\
\hline & 2 & 3 & 2 & - & & & 7 & 2 & 0.939 & & & 10 & 2 & 0.931 \\
\hline & & & 3 & - & & & & 3 & 0.995 & & & & 3 & - \\
\hline & & & 4 & 1 & & & & 4 & 0.985 & & & & 4 & - \\
\hline & & & 5 & 1 & & & & 5 & 0.998 & & & & 5 & 0.994 \\
\hline & & & 6 & 1 & & & & 6 & 0.997 & & & & 6 & - \\
\hline & & 4 & 2 & - & & & 8 & 2 & 0.924 & & & 11 & 2 & 0.994 \\
\hline & & & 3 & 0.964 & & & & 3 & 0.999 & & & & 3 & - \\
\hline & & & 4 & 0.983 & & & & 4 & 0.996 & & & & 4 & - \\
\hline & & & 5 & 1 & & & & 5 & 1 & & & & 5 & - \\
\hline & & & 6 & 0.996 & & & & 6 & 0.995 & & & & 6 & - \\
\hline & & 5 & 2 & 1 & & & 9 & 2 & 0.920 & & 3 & 7 & 2 & - \\
\hline & & & 3 & 0.921 & & & & 3 & 0.997 & & & & 3 & 1 \\
\hline & & & 4 & 0.958 & & & & 4 & 0.935 & & & & 4 & 0.997 \\
\hline & & & 5 & 0.997 & & & & 5 & 1 & & & & 5 & 0.997 \\
\hline & & & 6 & 0.990 & & & & 6 & 0.997 & & & & 6 & 0.994 \\
\hline & & 6 & 2 & 0.964 & & & 10 & 2 & 0.929 & & & 8 & 2 & - \\
\hline & & & 3 & - & & & & 3 & 0.996 & & & & 3 & 0.879 \\
\hline & & & 4 & 0.934 & & & & 4 & - & & & & 4 & 0.985 \\
\hline & & & 5 & 0.996 & & & & 5 & 0.998 & & & & 5 & 0.992 \\
\hline & & & 6 & 0.890 & & & & 6 & - & & & & 6 & - \\
\hline & & 7 & 2 & - & & & 11 & 2 & - & 3 & 1 & 10 & 2 & 1 \\
\hline & & & 3 & - & & & & 3 & 0.996 & & 2 & 10 & 2 & - \\
\hline & & & 4 & - & & & & 4 & - & & & & 3 & 1 \\
\hline & & & 5 & 0.995 & & & & 5 & 0.998 & & & 11 & 2 & 0.995 \\
\hline & & & 6 & 0.979 & & & & 6 & 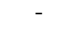 & & & & & \\
\hline
\end{tabular}


an equivalent-estimation two-levels split-split-plot design, the OLS-GLS equivalence has not yet been studied systematically in this context.

\section{Acknowledgment}

The first author of this article was sponsored by a project funded by the Research Foundation Flanders and by the Association of Scientific Authors VEWA.

\section{References}

Bingham, D. R., Schoen, E. D. and Sitter, R. R. (2004). Designing fractional factorial split-plot experiments with few whole-plot factors, Journal of the Royal Statistical Society: Series C 53: 325-339. Corrigendum, 54, 955-958.

Bingham, D. R. and Sitter, R. R. (1999). Minimum-aberration two-level fractional factorial split-plot designs, Technometrics 41: 62-70.

Goos, P. (2002). The Optimal Design of Blocked and Split-plot Experiments, New York: Springer.

Goos, P. (2006). Optimal versus orthogonal and equivalent-estimation design of blocked and split-plot experiments, Statistica Neerlandica 60: 361-378.

Goos, P. and Vandebroek, M. (2001). Optimal split-plot designs, Journal of Quality Technology 33: $436-450$.

Goos, P. and Vandebroek, M. (2003). D-optimal split-plot designs with given numbers and sizes of whole plots, Technometrics 45: 235-245.

Goos, P. and Vandebroek, M. (2004). Outperforming completely randomized designs, Journal of Quality Technology 36: 12-26.

Huang, P., Chen, D. and Voelkel, J. (1998). Minimum-aberration two-level split-plot designs, Technometrics 40: 314-326.

Jones, B. and Goos, P. (2007). A candidate-set-free algorithm for generating D-optimal split-plot designs, Journal of the Royal Statistical Society: Series C 56: 347-364.

Jones, B. and Goos, P. (2009). D-optimal design of split-split-plot experiments, Biometrika 96: $67-82$.

Jones, B. and Nachtsheim, C. J. (2009). Split-plot designs: What, why, and how, Journal of Quality Technology 41: 340-361.

Kulahci, M. and Bisgaard, S. (2005). The use of Plackett-Burman designs to construct split-plot designs, Technometrics 47: 495-501. 
Letsinger, J. D., Myers, R. H. and Lentner, M. (1996). Response surface methods for birandomization structures, Journal of Quality Technology 28: 381-397.

McElroy, F. W. (1967). A necessary and sufficient condition that ordinary least squares estimators be best linear unbiased, Journal of the American Statistical Association 62: 1302-1304.

Parker, P. A., Kowalski, S. M. and Vining, G. G. (2006). Classes of split-plot response surface designs for equivalent estimation, Quality and Reliability Engineering International 22: 291305.

Parker, P. A., Kowalski, S. M. and Vining, G. G. (2007a). Construction of balanced equivalent estimation second-order split-plot designs, Technometrics 49: 56-65.

Parker, P. A., Kowalski, S. M. and Vining, G. G. (2007b). Unbalanced and minimal point equivalent estimation second-order split-plot designs, Journal of Quality Technology 39: 376388.

Trinca, L. A. and Gilmour, S. G. (2001). Multi-stratum response surface designs, Technometrics 43: 25-33.

Vining, G. G., Kowalski, S. M. and Montgomery, D. C. (2005). Response surface designs within a split-plot structure, Journal of Quality Technology 37: 115-129. 